Published in final edited form as:

Curr Psychiatry Rep. 2013 February ; 15(2): 337. doi:10.1007/s11920-012-0337-0.

\title{
The Gut Microbiome: A New Frontier in Autism Research
}

\author{
Jennifer G. Mulle ${ }^{1,4}$, William G. Sharp ${ }^{2,3}$, and Joseph F. Cubells $\mathbf{s}^{4,5}$ \\ ${ }^{1}$ Department of Epidemiology, Emory University Rollins School of Public Health \\ ${ }^{2}$ Department of Pediatrics, Emory University School of Medicine \\ ${ }^{3}$ Marcus Autism Center, Children's Healthcare of Atlanta \\ ${ }^{4}$ Department of Human Genetics, Emory University School of Medicine \\ ${ }^{5}$ Emory Autism Center, Department of Psychiatry and Behavioral Sciences, Emory University \\ School of Medicine
}

\begin{abstract}
The human gut harbors a complex community of microbes that profoundly influence many aspects of growth and development, including development of the nervous system. Advances in highthroughput DNA sequencing methods have led to rapidly expanding knowledge about this gut microbiome. Here, we review fundamental emerging data on the human gut microbiome, with a focus on potential interactions between the microbiome and autism spectrum disorders (ASD) and consider research on atypical patterns of feeding and nutrition in ASD and how they might interact with the microbiome. Finally we selectively survey results from studies in rodents on the impact of the microbiome on neurobehavioral development. The evidence reviewed here suggests that a deeper understanding of the gut microbiome could open up new avenues of research on ASD, including potential novel treatment strategies.
\end{abstract}

\section{Keywords}

Gut microbiome; Nervous system; Behavior; Autism; Autism spectrum disorders; ASDs; Feeding; Nutrition; Dietary intake; Animal studies; Neurobehavioral development; Genetic disorders; Psychiatry

\section{Introduction}

We coexist with vast populations of microbial species that make a host out of the human body. It has been estimated that up to 100 trillion microbial cells make a home out of us ${ }^{1}$, and likely outnumber human body cells by an order of magnitude ${ }^{2}$, leading some to term the human microbiome our "second genome." 3 Increasingly, it has become clear that these constellations of microbial species are a partner in homeostasis, and when the balance is tipped away from the healthy microbiome there can be a negative outcome on human health ${ }^{4}$. This new appreciation has led to formal efforts to identify comprehensively all components of the microbiome. The Human Microbiome Project (HMP) was launched by

Corresponding author: Jennifer G. Mulle, PhD, Department of Epidemiology, Mailstop 1518-002-3BB, Emory University School of Public Health, Atlanta, GA 30322, Tel: +1 404-727-3042, Fax: +1 404-727-3949, jmulle@emory.edu.

Disclosure J. G. Mulle: research support from the National Institutes of Health (NIH) and consultant to the Centers for Disease Control and Prevention; W. G. Sharp: research support from the Children's Healthcare of Atlanta Trust Pilot Award; J. F. Cubells: research support from NIH, Roche, Seaside Therapeutics, and Biomarin and consultant to Abbott Laboratories, Novartis, Barnes and Thornberg, LLP, and the University of Nebraska. 
the NIH in 2007 to establish community resources, including a reference set of sequence data along with bioinformatics pipelines and standards for analysis ${ }^{5}$. The ultimate goal of the HMP is to understand how this "forgotten organ" functions to establish and maintain a healthy state, and whether the microbiome can be manipulated to favor long-term desirable health outcomes ${ }^{6}$.

\section{Progress in Understanding the Gut Microbiome}

Discrete populations of microbes reside on all surfaces exposed to the environment, including the skin, mouth, nasal cavity, and vagina, but it is in the gut that the most abundant and diverse community is found ${ }^{7}$. It has long been known that bacteria comprise a substantial component of human stool, with up to $55 \%$ of the biomass estimated to be due to bacteria $^{8}$. Up until the last decade, investigation of the gut microbiome was based upon conventional culture-based microbiology techniques ${ }^{9}$. Though tremendous strides were made using these methodologies, they can be laborious, expensive, and most importantly, subject to biased interpretation from species which are in low abundance, or cannot be cultured, affecting possibly $30-50 \%$ of species ${ }^{10-12}$. While this seems a substantial fraction, it is a rosier picture than soil or sea, for example, where $1 \%$ or less of bacterial species can be cultured in the lab. Enter culture-independent methods or "metagenomics," via shotgun sequencing, a method similar to that used to sequence the human genome, in which relatively short fragments of DNA are sequenced, and then assembled based on overlaps in sequence at the ends of the fragments ${ }^{13}$. Until recently, these methods were time-consuming and expensive, and were difficult to apply to large numbers of samples. The advent of nextgeneration sequencing strategies that have decreased costs of sequencing while simultaneously increasing throughput, have enabled a revolution in the detection of new microbial species in soil, sea, and in the microbiomes of many species including humans. A particular advantage of these approaches is that they account for microbial species that cannot be cultured in a laboratory setting. Another major scientific advantage of metagenomic analysis is that phylogenetic relationships can be readily inferred from comparisons of DNA sequence, whereas older approaches to classification of bacterial species, which relied on phenotypic characteristics such as gross morphology (e.g., rods vs. cocci) or nutritional requirements for growth in culture, did not provide systematic information on evolutionary relationships among species ${ }^{14}$. One of the first applications of metagenomic methods to identify new microorganisms was in filtered sea water from the Sargasso Sea, where 148 previously-unknown bacterial phyla were newly identified ${ }^{15}$. These data provided a rationale for systematic culture-independent efforts aimed toward identifying components of the human microbiome.

The distal gut microbiome is typically assayed via stool because of the non-invasive route of collection, and have provided important insights into the distal gut microbiome composition and function. However, it is important to recognize that adherent microbiota residing on mucosal surfaces of the gut may be vastly different from those present in fecal material; furthermore, different compartments of the gut may be locally colonized by distinct communities of bacteria ${ }^{16}$. The procedures required to sample adherent species are invasive, expensive and impractical for a typical research study. Nevertheless, while existing data on the distal gut microbiome should be interpreted with this limitation in mind, there is much to be learned even from the partial information available from metagenomic analysis of stool.

Recent metagenomics data have confirmed the high bacterial composition of stool, with $>90 \%$ of sequence data belonging to bacteria ${ }^{17}$. The gut microbiome is dominated by bacteria belonging two phyla, Firmicutes or Bacteriodetes ${ }^{17}$. Phylogenetic analysis of metagenomics data from 22 individuals revealed three orthogonal clustures of data, termed "enterotypes," driven largely by the presence of one of three genera (Bacteroides, Prevotella, 
Ruminococcus $)^{17}$. A subsequent paper which coupled metagenomic data with nutrient intake found enterotypes were clustered according to long-term dietary intake, with enterotypes remaining stable over up to 10 days, even in the presence of substantial dietary changes via a controlled feeing study ${ }^{18}$. In addition to diet, age, and other environmental factors, host genetics are likely to play a role in establishing and maintaining the enterotype. Well-designed prospective studies will be required to derive the contribution of these primary factors influencing enterotype formation.

While most studies are done in adults, the few studies done in infants and children have revealed astonishing insights into the developing gut microbiome. Human babies are believed to be born with sterile gastro-intestinal tracts ${ }^{19,20}$ but immediately upon birth colonization of the gut by microbes begins. Mode of birth (vaginal delivery compared to cesarean section) has been shown to be the primary initial influence of the developing infant microbiome ${ }^{21}$. Additionally, breast-fed vs formula-fed infants have very different trajectories of gut microbiome development ${ }^{22}$ (see below for expansion of this theme). Over the first years of life the gut microbiome is changing and remodeling, ultimately resembling an adult gut microbiome by year $3^{19}$. This suggests there is a "core microbiome" that is the hallmark of a healthy individual. In a large metagenomic sequencing study of stool samples from 124 individuals, the number of distinct microbial genes was estimated to be 3.3 million $^{23}$; to put this number in context, it is widely believed that the entire human genome contains about 30,000 protein-coding genes. Thus, humans are harboring functional genes that likely complement our existing biology, though these functions are kept annexed in our distal gut. This implies the gut microbiome serves a specific, and important, purpose, begging the question: what function does the gut microbome serve?

\section{Functions of the Gut Microbiome}

\section{Nutrition}

It has been proposed that the gut microbiome functions in nutrient intake. As noted above, the collective genetic complement of the gut microbiome is $\sim 150 \mathrm{x}$ greater than the human genome $^{23}$. It is conspicuous that the human genome lacks enzymes to degrade many plant polysaccharides, such as those containing pectin, arabinose, and xylan; in contrast, the distal gut microbiome contains more than 80 different glycoside hydrolase families ${ }^{2}$. Additional evidence for this functionality comes from infants, where the developing gut microbiome is enriched for genes involved in lactate utilization, consistent with lactose as an energy source from breast milk or formula ${ }^{19}$. In this way, the gut microbiome may have evolved as an adaptation to allow extraction of maximal energy from food sources. In fact, it has been estimated that up to $10 \%$ of available calories in a western diet come from microbial fermentation of carbohydrates in the distal gut ${ }^{24,25}$, providing an evolutionary rationale for the continued maintenance of a rich and extensive gut microbial community ${ }^{26}$.

\section{Immunologic Development}

The gut microbiome may also serve as a tuning fork for the developing immune system. There is a vast and complex interaction between the gastrointestinal mucosa and the commensal bacteria, mediated largely by components of the innate immune system ${ }^{27}$. The intestinal immune system must walk a fine line, learning to tolerate helpful bacteria while retaining the ability to mount a response to pathogenic species ${ }^{3}$. How this is achieved is not yet clear, but it is likely that symbiotic bacteria have evolved to survive the harsh conditions of the intestinal lumen, perhaps by accumulating functions that allow coexistence with the host immune system. Data are emerging for this hypothesis, supporting defined roles for specific bacterial species. For example, in mice, it has been shown that the intestinal T-cell repertoire is strongly influenced by segmented filamentous bacteria (SFB), a species related 
to Clostridia. Without the presence of SFB, intestinal T-cells fail to develop ${ }^{28}$. It has also been shown in mice that exposure to a specific polysaccharide produced by Bacteriodes fragilis interacts with the host immune system to induce proper T-cell development, correct Th1/Th2 imbalances, and direct appropriate development of the spleen and other secondary lymphoid tissues ${ }^{29}$.

\section{Pathology}

We are just beginning to understand what comprises a "normal" gut microbiome, but there are already associations between unhealthy states and abnormal or imbalanced gut microbiota. For example, it has been shown that in obese individuals, the gut microbiome is characterized by a loss of diversity ${ }^{30}$. Aberrations in the gut microbiome have been shown to play a role in inflammatory bowel disease ${ }^{23}$. Specific bacterial metabolites are associated with an increased risk for heart disease ${ }^{31}$. And in autism spectrum disorders (ASD), there are hints that the gut microbiome may play a role. Studies of fecal DNA extracts have found Clostridium or Desulfovibrio clusters over-represented in children with gastrointestinal complaints and ASD as compared to children with similar GI complaints but typical neurobehavioral development ${ }^{32-34}$. Furthermore, clinical improvement has been reported anecdotally in children with ASD who develop fever ${ }^{35}$, receive oral antibiotics ${ }^{36}$, or ingest probiotics $^{37}$, all of which are likely to alter the gut microflora. While these initial studies are promising, they suffer from small sample sizes, in part due to the laborious, conventional, culture-dependent techniques used to identify bacterial species. Fortunately, the availability of metagenomic technology, which obviates culture-dependent methods, can provide a comprehensive, unbiased estimate of bacterial species, and is amenable to application in large case-control studies. Such studies could provide data unambiguously to implicate or to exonerate the gut microbiome as a player in ASD.

\section{Feeding Practices and the Gut Microbiome}

Diet plays a critical function in the establishment, maturation, and maintenance of microbial diversity in the gut ecosystem. Beginning at birth, feeding practices help modulate intestinal colonization, with breastfed infants differing in the composition and volume of gut microbial species compared to formula-fed infants ${ }^{38}$. In addition to providing optimal nutrition, breast milk represents an important source of protective factors largely absent from formula, including commensal bacteria, that aide in the development of the gastrointestinal (GI) tract, pancreas/endocrine system, and related mucosal defenses ${ }^{39}$. Evidence suggests human milk is an important source of lactic acid bacteria (most notably Lactobacillus) and bifidobacteria (including specific Bifidobacterium species) for newborns. High prevalence of Bifidobacterium and Lactobacillus is a biological marker of healthy gut microbiota in breast-fed infants, with both types of microbes serving important probiotic functions in the gut, including inhibition of pathogenic bacteria through competitive exclusion and/or production of antimicrobial agents ${ }^{38,40,41}$.

Colonization of the human gut involves a gradual, successive process influenced by diet and other life events, with Bifidobacterium and Lactobacillus emerging at temporarily distinct points in the gut of healthy breastfed infants. Bifidobacterium appears shortly after birth and, within weeks, represents the dominant microorganism in the gut; Lactobacillus, in contrast, emerges over a period of months ${ }^{42}$. Research also suggests individual species and/or strains of the same bacterial genus may exert different effects on the developing immune system. For example, Bifidobacterium longum, the strain most often associated with human breast milk, appears to have strain-specific effects on both systemic and intestinal immunity, while Bifidobacterium breve has little or no impact on the immune function ${ }^{43}$. Bifidobacterium adolescentis has been found to be more prevalent in allergic infants manifesting atopic eczema, while Bifidobacterium bifidum is most often colonized in healthy, breastfed 
infants ${ }^{44}$. Deviations in neonatal gut microflora, including more Clostridia and fewer Bifidobacterium, have also been shown to preceed the later development of atopic sensitization and subsequent atopic disease, providing additional evidence regarding the potential role of gut colonization and commensal microflora in modulating immunity, including regulating hypersensitivity to food and/or other environmental elements ${ }^{45}$.

Based on the critical role of human milk in providing optimal nutrition while concurrently assisting with colonization of the gut microbiome, it is not surprising breast-feeding is associated with numerous health benefits during infancy, including higher resistance to illness and lower rates of gastroenteritis, respiratory infections, otitis media, urinary tract infections, infectious diarrhea, and necrotising enterocolitis ${ }^{39,46}$. Breast-feeding also provides long-term protection against inflammatory bowel disease (IBD), diabetes, and obesity later in life ${ }^{39}$ and provisional evidence suggests that these health benefits may, in part, be related to the establishment of a healthy gut microbiome. Microbial dysbiosis is associated with chronic disease in both infancy and adulthood, including distinct patterns of predominant bacteria phyla linked with IBD and diabetes in adults, as well as necrotizing enterocolitis in infants ${ }^{47}$. Further, recent evidence suggests that gut microbiota may play a causal role in disease pathology, with traits of complex diseases (e.g., adiposity; metabolic syndrome) demonstrated to transfer through gut microbiota in animal models ${ }^{48,49}$. Microbial species transmitted through human breast milk may also protect against crying and fussiness during early infancy, with specific biological markers including early colonization of Bifidobacterium and greater prevalence on Lactobacillus ${ }^{42}$. In turn, deviations in the colonization and resulting gut ecosystem (including less diverse fecal microflora) have been posited to underlie infant colic, a condition previously attributed to non-organic factors ${ }^{50,51}$.

Breast-feeding also enhances cognitive functioning, as well as educational and developmental outcomes. Benefits include enhanced performance on standardized tests, teacher ratings, and academic achievement, which extend throughout childhood into early adulthood $^{52}$. Evidence also suggests suboptimal breast-feeding practices, including late initiation of breast-feeding and shorter duration of exclusive breast-feeding, may increase the risk for $\mathrm{ASD}^{53}$. This relationship follows a dose-response pattern, with the risk for ASD decreasing with more prolonged periods of exclusive breast-feeding for the first 6 month and continued breast-feeding through 2 years of life. Breast-feeding also appears to be less prevalent and, when present, occurs for a much shorter duration in ASD. Children with ASD are significantly more likely to be weaned by the end of the first month of life compared to typically peers ${ }^{54}$ and absence of breast-feeding is more prominent in this population ${ }^{55}$. Factors contributing to suboptimal breast feeding in ASD have yet to be identified, although the cause is likely multi-factorial. One possibility is that mothers of children with ASD terminate or fail to initiate breast feeding due to difficulty with the child's engagement in the feeding process. Alternatively, temperamental or behavioral issues associated with ASD may make breastfeeding more challenging in this population. Clearly, more research is needed in this area. There is, however, sufficient evidence indicating the ASD population is at-risk for reduced exposure to breast-milk, including both the nutritional benefits and commensal bacteria transmitted through this method of feeding. Deviations in feeding practices in ASD, in turn, are likely to lead to atypical gut colonization and microbial dysbiosis, which helps explain differences in the microbial ecosystem detected in ASD while also raising new questions regarding the possible contribution of organic-factors in the prevalence of feeding problems in this population.

\section{Feeding Problems and Dietary Intake in ASD}

Estimates suggest upwards of $90 \%$ of children with ASD experience some type of feeding related concern ${ }^{56}$. Food selectivity (i.e., only eating a narrow variety of foods by type, 
texture, and/or presentation) represents the most pervasive feeding issue associated with ASD, typically in the form of strong preferences for starches, snack and processed foods while rejecting most (if not all) fruits, vegetables, and/or proteins ${ }^{57,58}$. Emerging evidence suggests atypical patterns of intake in ASD may increase the risk for nutritional and/or related medical issues in this population. Possible detrimental outcomes include poor bone growth $^{59}$ and chronic vitamin and mineral deficiencies ${ }^{60,61}$. High consumption of fats and snacks may also portend increased long-term risk for chronic health conditions among adults with ASD, with diets high in saturated fat associated with diseases such as cardiovascular disease and cancer in the general population ${ }^{62,63}$. Such high prevalence combined with possible long-term medical sequelae intensifies the need to indentify the cause, long-term impact, and remediation of atypical feeding in ASD.

Etiological factors contributing to feeding problems in ASD remain elusive. While organic issues (e.g., food allergies; gastroesophageal reflux) often co-occur with the emergence and/ or maintenance of feeding concerns in children without ASD, past reports have yet to identify a clear GI etiology to account for the pattern and prevalence of food selectivity in the ASD population ${ }^{64}$. At this time, evidence a. suggests a similar topography and prevalence of GI conditions among children with and without ASD and b. there is no GI disturbance (e.g., "autistic enterocolitis") unique to the diagnosis ${ }^{65}$. Although higher rates of constipation and encopresis (as a consequence of constipation) occur in ASD, these concerns have a strong behavioral component, suggesting a neurobehavioral, rather than primary organic etiology ${ }^{66}$. Various environmental/behavioral factors have been posited to account for feeding problems in ASD, including maladaptive patterns of reinforcement and communications difficulties ${ }^{56,67}$. It has also been suggested that food selectivity in ASD is due to behavioral rigidity, representing an additional manifestation of this core diagnostic feature ${ }^{68}$. Available theories, however, do not explain the pattern of dietary preference observed in ASD, including why this population gravitates toward consuming fats, snacks, and other processed foods while avoiding vegetables and fruits.

As noted above, a major function of a healthy gut microbiome (particularly members of the phylum Bacteroidetes) is to assist in the breakdown of complex plant polysaccharides and other "nondigestible" dietary matter, with a diet high in plant material supporting a microbial community that promotes digestion and supports overall host health ${ }^{19}$. Therefore, it is possible that deviations in the establishment and maintenance of the gut microbiome in ASD may lead to pain and discomfort (e.g., inflammation; bloating; increased flatulence) due to difficulty in digesting plant based foods ${ }^{69}$. Food selectivity, however, may represent the only prominent sign of GI distress in ASD due to communication difficulties inherent to the condition, with this population refusing foods that rely on gut microbiota for processing and, thus, avoiding pain associated with difficulties with digestion. This hypothesis is consistent with research indicating many longstanding feeding problems involve learned behaviors whose function is to escape unpleasant feeding experiences ${ }^{70}$. Atypical intake combined with deficient digestion and absorption may further perpetuate this process by underutilizing and depleting the micorbial community of Bacteroidetes present in the healthy intestine while concurrently promoting competitive growth of less beneficial bacterial phylotypes ${ }^{69}$.

Although research is needed to elucidate the relationship between feeding problems, dietary patterns and gut dysbiosis in ASD, it seems plausible that interventions aimed at restoring the microbial balance in the gut may improve behaviors (e.g., irritability, anxiety, and social withdrawal) documented to occur more frequently among a subgroup of individuals with ASD and GI symptoms ${ }^{71}$. Indeed, probiotics (i.e., microorganisms ingested through food or supplement consisting primarily of lactic acid-producing bacteria, such as lactobacilli, lactococci, bifidobacteria) have been shown to improve symptoms of irritable bowel 
syndrome, such as bloating, abdominal pain, and flatulence ${ }^{72}$, and suggested as a possible intervention to improve behavioral issues associated with GI discomfort in $\mathrm{ASD}^{37}$. It may also help explain anecdotal reports of improved functioning following dietary changes among certain children with ASD, which have helped propagate interest regarding the use of dietary manipulation (e.g., gluten and/or casein free, GFCF diet) in this population.

Studies of humans thus far have provided tantalizing observations on potential differences in the composition of gut microbiota associated with complex behavioral conditions such as obesity and ASD. Those observations certainly require continued investigation. However, ethical considerations preclude many experimental approaches to understanding the role of the microbiome in neurobehavioral development. Thus, studies of other mammalian species complement studies of humans in important ways. Emerging studies of rodents have begun to explore how differences in gut microbiota may influence neuro-behavioral development.

\section{Animal Studies on the Gut Microbiome and Neural Development}

Among the most important tools in the experimental biology of the microbiome are socalled gnotobiotic animals_-organisms that harbor a known set of microbial species. Germfree (GF) mice, produced by delivering neonatal animals by Cesarean section in sterile conditions and immediately transferring them to a completely sterile environment where they are foster raised, represent one end of the gnotobiotic spectrum and have been particularly useful experimental models for studying the influence of microbes on the development of the immune and nervous systems. Another class of gnotobiotic mice are initially GF mice raised from early post-natal life in the presence of commensal microbial species but in the specific absence of pathogenic organisms: specific-pathogen-free (SPF) mice. Other types of gnotobiotic mice include those raised from birth, or reconstituted at later post-natal times, with a broader, but still explicitly defined, microbial milieu, as well as mono-associated varieties, colonized by specific single microbial species.

Studies of gnotobiotic mice have contributed to our emerging understanding of the "braingut-microbiota axis" $73 * *$. The gut and nervous systems communicate via neural mechanisms (e.g., parasympathetic innervation by the vagus nerve, as well as sympathetic innervation from multiple levels of the sympathetic chain; reviewed by ${ }^{74}$ ), and endocrine pathways such as the hypothalamic-pituitary-adrenal (HPA) axis ${ }^{75}$. In addition, immunemediated mechanisms play important roles in the way the gut and brain influence each other's function and development ${ }^{76}$. GI microbes thus influence brain function and behavior through several sets of complex pathways.

Several research groups have reported that GF mice display behavioral differences at baseline as compared to non-GF comparison animals. Thus, Diaz Heijtz and colleagues 77 showed that GF mice emitted more locomotor behavior and rearing in an open field, as well as greater amounts of time spent in the lighted portion of a light-dark paradigm, or the open arms of an elevated plus maze, as compared to SPF mice of the same outbred strain, which the authors interpreted as reduced "anxiety-like" behavior in the GF mice. Remarkably, the authors also showed that GF mice colonized at 3 weeks of age with SPF microbiota later showed identical behaviors to SPF mice as adults, but such colonization at 6 weeks did not restore SPF-like behavior in adulthood, suggesting the hypothesis that there are critical periods during which commensal flora influence neurobehavioral development. In that same study, GF mice exhibited increased metabolic turnover of dopamine, norepinephrine and serotonin, as well as brain-region-specific differences in expression of mRNA encoding a variety of gene products including brain-derived neurotrophic factor (BDNF). GF mice also showed differences in levels of several synaptic proteins in extracts of cortex, striatum and hippocampus. The study provided strong evidence supporting the hypothesis that the 
developing gut microbiome exerts important "programming" influences on adult behavioral responses, as well as adult neurochemistry and brain gene expression, and that such effects may occur during critical periods.

Clarke et al. ${ }^{78}$ focused on the interaction of sex and the gut microbiome, examining stress responses, the hippocampus and the serotonergic system as neuro-behavioral endpoints in GF mice and conventionally colonized (CC) mice stratified by sex. GF mice of both sexes exhibited greater release of corticosterone in response to a novel environment. GF male, but not GF female mice showed diminished expression of mRNA encoding BDNF, and greater levels of serotonin, in the hippocampus compared to CC mice of the same sex. Male GF mice also showed higher levels of plasma tryptophan and serotonin, and a diminished ratio of kynurenine to tryptophan suggestive of a shunting of tryptophan metabolism away from the (usually predominant) kynurenine pathway toward serotonin biosynthesis. Colonization of GF male mice at 3 weeks of age reversed the differences in plasma serotonin-related measures, but not in hippocampal serotonin levels. Thus, while this study did not convincingly demonstrate systematic relationships among the many parameters they examined, it provides important evidence that specific effects of the microbiome on neural development can vary with sex, and that some, but not all influences of GF status in early life can be reversed by later colonization with conventional gut flora.

Sudo and colleagues ${ }^{75}$ showed that GF mice exhibit greater release of adrenocorticotrophic hormone (ACTH) and corticosterone in response to restraint stress than SPF mice. They extended this observation to demonstrate that the species composition of the microbiome differentially alters HPA function. Mice colonized neonatally only with Bifidobacterium infantis, a commensal species that is part of the normal gut flora, exhibited restraint-stressinduced ACTH and corticosterone responses comparable to those of SPF mice, whereas those colonized at birth with an enteropathogenic (i.e., disease-causing) strain of Escherichia coli showed a more dramatic stress-hormone response than GF mice. Thus, not only does neonatal colonization with gut micro-organisms alter the responsiveness of the adult HPA axis to stress, but different microbial species can affect such development in different ways. The influence of the enteropathogenic E. coli on HPA axis development appeared to depend on its ability to invade gut mucosal cells, because colonization of GF mice with $\Delta$ Tir, a mutant enteropathogenic E. coli strain that lacks the capacity to translocate across the cell membrane, failed to produce the same effect as the translocation-competent strain. Finally, this study demonstrated that colonization at different time points in early post-natal life had different influences on HPA responsiveness in later life, supporting the hypothesis already discussed that there may be critical periods during which the developing host is more or less sensitive to the programming influence of gut microbes.

Studies of gnotobiotic rodents during adulthood have demonstrated that specific interventions can alter behavioral and nuerochemical phenotypes. Non-absorbable antibiotics altered the relative abundance of microbial species in adult SPF mice, at the same time resulting in elevated exploratory behavior in light-dark and step-down paradigms ${ }^{79}$, as well as elevated levels of BDNF in the hippocampus and diminished levels in the amygdala. The behavioral effect of antibiotic treatment did not occur in GF mice, suggesting that a colonized gut was required for the effect. Testing of antibiotic-treated SPF mice after a twoweek washout period during which the microbiome reverted to a pattern similar to that observed in untreated animals also failed to yield the behavioral differences observed immediately after antibiotic treatment, suggesting that the behavioral differences were reversible.

Different strains of mice are well-known to display different behavioral profiles. For example, Balb/C mice tend to exhibit higher levels of anxiety-like behaviors (e.g., low 
frequencies of locomotion into the middle portion of an open field) than other strains ${ }^{80}$. The study just cited on the effects of non-absorbable antibiotics on behavior ${ }^{79}$, also showed that fecal DNA sequence profiles (assessed by denaturing gradient gel electrophoresis, which allows profiling of relative abundance of different sequences, but does not provide specific base-pair sequence data) differ between strains of mice maintained in SPF conditions. Remarkably, they then demonstrated that colonization of GF mice by fecal transplantation from Balb/c mice resulted in different behavioral outcomes, and hippocampal (but not amygdala) levels of BDNF, than fecal transplants from NIH-Swiss mice. Thus, the data suggest strongly that gut microbiota can influence behavior and brain chemistry, and that the genetic characteristics of the host interact with the microbiome to determine developmental trajectories.

Subtherapeutic antibiotic treatment (STAT) is ubiquitous in animal husbandry, based on the longstanding observation that including low doses of a variety of antibiotics in the feed of animals enhances weight-gain (and hence profits in an industry where animals are sold by weight). A recent study of STAT in laboratory mice has provided strong evidence that alterations in the gut microbiome mediate many important aspects of this weight-gain enhancing effect. Treatment of mice from weaning with several different STAT regimens (administered in the drinking water) resulted in no overall differences in weight gain after 7 weeks, but did result in increases in fat mass and bone-mineral density in the treated animals. The overall gut-microbial "census" was similar in controls and treated animals (meaning the total microbial biomass did not differ substantially among groups), but there were significant differences in relative proportions of microbial taxa, with Firmicutes to Bacteroides ratios greater in the treated animals. Analysis of specific microbial metabolic genes showed greater abundance of genes encoding specific enzymes involved in shortchain fatty acid synthesis, indicating a shift in the metabolic capacity of the microbiome due to STAT, which was accompanied by corresponding changes in concentrations of ratios of specific short-chain fatty acids. Microarray analysis of host-liver gene expression revealed an increase in expression of genes encoding proteins important in fatty-acid and triglyceride synthesis, strongly suggesting that the microbiome influences liver function related to adiposity by altering exposure of hepatocytes to nutrients in the portal circulation. Interestingly, there were no differences observed in caloric consumption of the mice in different groups, but analysis of fecal caloric content showed that the STAT animals extracted more calories from the food they consumed. This study suggests that early manipulation of the gut microbiome produces longterm differences in critical metabolic processes contributing to growth and development. Despite the similarity in caloric consumption across groups, the study did not examine feeding behavior per se, in terms of parameters such as frequency and size of meals. Another aspect of feeding not amenable to analysis in an experiment on chow-fed rodents is food preferences. Future studies should examine such variables, as they might reveal important relationships between the microbiome and feeding behavior relevant to ASD and other behavioral disorders.

\section{Conclusion}

The data just reviewed strongly suggests that further research on the relationships among the gut microbiome, the development and function of the nervous system, and behavior will be fruitful. The field is at a stage where more questions than answers are being generated. Difficulties with feeding and gastro-intestinal function are virtually ubiquitous in ASD, as has been reviewed here. That clinical observation, together with evidence just reviewed supporting associations between gut microbial population profiles and ASD, and the data from research on rodents demonstrating myriad ways in which the gut microbiome influences neurobehavioral development, combine to suggest that further research on the trajectory of microbiome development in children at risk for ASD will be fruitful. The 
possibility of specific interventions to alter microbiome development opens up the prospect of a range of novel therapeutic approaches such as probiotic, antibiotic or dietary manipulations that may offer hope to patients and families living with ASD.

\section{References}

Papers of particular interest, published recently, have been highlighted as:

- Of importance

•• Of outstanding importance

1. Whitman WB, Coleman DC, Wiebe WJ. Prokaryotes: the unseen majority. Proceedings of the National Academy of Sciences of the United States of America. 1998; 95:6578-6583. [PubMed: 9618454]

2. Gill SR, et al. Metagenomic analysis of the human distal gut microbiome. Science. 2006; 312:13551359. [PubMed: 16741115]

3. Zhu B, Wang X, Li L. Human gut microbiome: the second genome of human body. Protein \& cell. 2010; 1:718-725. [PubMed: 21203913]

4. Clemente JC, Ursell LK, Parfrey LW, Knight R. The impact of the gut microbiota on human health: an integrative view. Cell. 2012; 148:1258-1270. [PubMed: 22424233]

5. Peterson J, et al. The NIH Human Microbiome Project. Genome research. 2009; 19:2317-2323. [PubMed: 19819907]

6. O'Hara AM, Shanahan F. The gut flora as a forgotten organ. EMBO reports. 2006; 7:688-693. [PubMed: 16819463]

**7. A framework for human microbiome research. Nature. 2012; 486:215-221. One of the initial publications from the Human Microbiome Project, showing the diversity of data contained in the microbiome from different body sites. [PubMed: 22699610]

8. Cummings JH, Macfarlane GT. Role of intestinal bacteria in nutrient metabolism. JPEN Journal of parenteral and enteral nutrition. 1997; 21:357-365. [PubMed: 9406136]

9. Staley JT, Konopka A. Measurement of in situ activities of nonphotosynthetic microorganisms in aquatic and terrestrial habitats. Annual review of microbiology. 1985; 39:321-346.

10. Wade W. Unculturable bacteria--the uncharacterized organisms that cause oral infections. Journal of the Royal Society of Medicine. 2002; 95:81-83. [PubMed: 11823550]

11. Duncan SH, Louis P, Flint HJ. Cultivable bacterial diversity from the human colon. Letters in applied microbiology. 2007; 44:343-350. [PubMed: 17397470]

12. Eckburg PB, et al. Diversity of the human intestinal microbial flora. Science. 2005; 308:16351638. [PubMed: 15831718]

13. Shendure J, Ji H. Next-generation DNA sequencing. Nature biotechnology. 2008; 26:1135-1145.

14. Pace NR. A molecular view of microbial diversity and the biosphere. Science. 1997; 276:734-740. [PubMed: 9115194]

15. Venter JC, et al. Environmental genome shotgun sequencing of the Sargasso Sea. Science. 2004; 304:66-74. [PubMed: 15001713]

16. Momozawa Y, Deffontaine V, Louis E, Medrano JF. Characterization of bacteria in biopsies of colon and stools by high throughput sequencing of the V2 region of bacterial 16S rRNA gene in human. PloS one. 2011; 6:e16952. [PubMed: 21347324]

**17. Arumugam M, et al. Enterotypes of the human gut microbiome. Nature. 2011; 473:174-180. The first paper with data to support the idea of characteristic gut microbiomes, classified as "enterotypes.". [PubMed: 21508958]

18. Wu GD, et al. Linking long-term dietary patterns with gut microbial enterotypes. Science. 2011; 334:105-108. [PubMed: 21885731]

*19. Koenig JE, et al. Succession of microbial consortia in the developing infant gut microbiome. Proceedings of the National Academy of Sciences of the United States of America. 2011; 108(Suppl 1):4578-4585. This study measured the developing infant gut microbiome by 
sampling stool from a single healthy infant 60 times, from birth to 2.5 years of age. [PubMed: 20668239]

20. Breitbart M, et al. Viral diversity and dynamics in an infant gut. Research in microbiology. 2008; 159:367-373. [PubMed: 18541415]

21. Dominguez-Bello MG, et al. Delivery mode shapes the acquisition and structure of the initial microbiota across multiple body habitats in newborns. Proceedings of the National Academy of Sciences of the United States of America. 2010; 107:11971-11975. [PubMed: 20566857]

*22. Schwartz S, et al. A metagenomic study of diet-dependent interaction between gut microbiota and host in infants reveals differences in immune response. Genome biology. 2012; 13:r32. This study compared both the developing gut microbiome and gene expression in 6 breastfed infants and 6 formula fed infants, finding systematic differences in the microbiome and in expression of genes relevant to immune system fucntion. [PubMed: 22546241]

23. Qin J, et al. A human gut microbial gene catalogue established by metagenomic sequencing. Nature. 2010; 464:59-65. [PubMed: 20203603]

24. Bergman EN. Energy contributions of volatile fatty acids from the gastrointestinal tract in various species. Physiological reviews. 1990; 70:567-590. [PubMed: 2181501]

25. Walter J, Ley R. The human gut microbiome: ecology and recent evolutionary changes. Annual review of microbiology. 2011; 65:411-429.

26. Chow J, Lee SM, Shen Y, Khosravi A, Mazmanian SK. Host-bacterial symbiosis in health and disease. Advances in immunology. 2010; 107:243-274. [PubMed: 21034976]

27. Kaplan JL, Shi HN, Walker WA. The role of microbes in developmental immunologic programming. Pediatric research. 2011; 69:465-472. [PubMed: 21364495]

28. Gaboriau-Routhiau V, et al. The key role of segmented filamentous bacteria in the coordinated maturation of gut helper T cell responses. Immunity. 2009; 31:677-689. [PubMed: 19833089]

29. Mazmanian SK, Liu CH, Tzianabos AO, Kasper DL. An immunomodulatory molecule of symbiotic bacteria directs maturation of the host immune system. Cell. 2005; 122:107-118. [PubMed: 16009137]

30. Turnbaugh PJ, et al. A core gut microbiome in obese and lean twins. Nature. 2009; 457:480-484. [PubMed: 19043404]

31. Wang Z, et al. Gut flora metabolism of phosphatidylcholine promotes cardiovascular disease. Nature. 2011; 472:57-63. [PubMed: 21475195]

32. Finegold SM, et al. Pyrosequencing study of fecal microflora of autistic and control children. Anaerobe. 2011; 16:444-453. [PubMed: 20603222]

33. Parracho HM, Bingham MO, Gibson GR, McCartney AL. Differences between the gut microflora of children with autistic spectrum disorders and that of healthy children. J Med Microbiol. 2005; 54:987-991. [PubMed: 16157555]

34. Song Y, Liu C, Finegold SM. Real-time PCR quantitation of clostridia in feces of autistic children. Appl Environ Microbiol. 2004; 70:6459-6465. [PubMed: 15528506]

35. Curran LK, et al. Behaviors associated with fever in children with autism spectrum disorders. Pediatrics. 2007; 120:e1386-1392. [PubMed: 18055656]

36. Sandler RH, et al. Short-term benefit from oral vancomycin treatment of regressive-onset autism. J Child Neurol. 2000; 15:429-435. [PubMed: 10921511]

37. Critchfield JW, van Hemert S, Ash M, Mulder L, Ashwood P. The potential role of probiotics in the management of childhood autism spectrum disorders. Gastroenterology research and practice. 2011; 2011:161358. [PubMed: 22114588]

38. Gueimonde M, Laitinen K, Salminen S, Isolauri E. Breast milk: a source of bifidobacteria for infant gut development and maturation? Neonatology. 2007; 92:64-66. [PubMed: 17596738]

39. Le Huerou-Luron I, Blat S, Boudry G. Breast- v. formula-feeding: impacts on the digestive tract and immediate and long-term health effects. Nutrition research reviews. 2010; 23:23-36. [PubMed: 20450531]

40. Martin R, Heilig GH, Zoetendal EG, Smidt H, Rodriguez JM. Diversity of the Lactobacillus group in breast milk and vagina of healthy women and potential role in the colonization of the infant gut. Journal of applied microbiology. 2007; 103:2638-2644. [PubMed: 18045446] 
41. Sinkiewicz, G.; Nordsröm, EA. European Society For Pediatric Research (ESPR). Vol. 58. Sienna, Italy: 2005. Occurrence of lactobacillus reuteri, lactobacilli, and bifidobacteria in human breast milk; p. 415Occurrence of lactobacillus reuteri, lactobacilli, and bifidobacteria in human breast milk

42. Partty A, Kalliomaki M, Endo A, Salminen S, Isolauri E. Compositional development of Bifidobacterium and Lactobacillus microbiota is linked with crying and fussing in early infancy. PloS one. 2012; 7:e32495. [PubMed: 22403665]

43. Menard O, Butel MJ, Gaboriau-Routhiau V, Waligora-Dupriet AJ. Gnotobiotic mouse immune response induced by Bifidobacterium sp. strains isolated from infants. Applied and environmental microbiology. 2008; 74:660-666. [PubMed: 18083875]

44. Ouwehand AC, et al. Differences in Bifidobacterium flora composition in allergic and healthy infants. The Journal of allergy and clinical immunology. 2001; 108:144-145. [PubMed: 11447399]

45. Kalliomaki M, et al. Distinct patterns of neonatal gut microflora in infants in whom atopy was and was not developing. The Journal of allergy and clinical immunology. 2001; 107:129-134. [PubMed: 11150002]

46. Bier JA, Oliver T, Ferguson AE, Vohr BR. Human milk improves cognitive and motor development of premature infants during infancy. Journal of human lactation: official journal of International Lactation Consultant Association. 2002; 18:361-367. [PubMed: 12449052]

47. Spor A, Koren O, Ley R. Unravelling the effects of the environment and host genotype on the gut microbiome. Nature reviews Microbiology. 2011; 9:279-290.

48. Turnbaugh PJ, Backhed F, Fulton L, Gordon JI. Diet-induced obesity is linked to marked but reversible alterations in the mouse distal gut microbiome. Cell host \& microbe. 2008; 3:213-223. [PubMed: 18407065]

49. Vijay-Kumar M, et al. Metabolic syndrome and altered gut microbiota in mice lacking Toll-like receptor 5. Science. 2010; 328:228-231. [PubMed: 20203013]

50. Rhoads JM, et al. Altered fecal microflora and increased fecal calprotectin in infants with colic. The Journal of pediatrics. 2009; 155:823-828. e821. [PubMed: 19628216]

51. Savino F, et al. Bacterial counts of intestinal Lactobacillus species in infants with colic. Pediatric allergy and immunology: official publication of the European Society of Pediatric Allergy and Immunology. 2005; 16:72-75. [PubMed: 15693915]

52. Horwood LJ, Fergusson DM. Breastfeeding and later cognitive and academic outcomes. Pediatrics. 1998; 101:E9. [PubMed: 9417173]

53. Al-Farsi YM, et al. Effect of suboptimal breast-feeding on occurrence of autism: a case-control study. Nutrition. 2012; 28:e27-32. [PubMed: 22541054]

54. Tanoue Y, Oda S. Weaning time of children with infantile autism. Journal of autism and developmental disorders. 1989; 19:425-434. [PubMed: 2793787]

55. Schultz ST, et al. Breastfeeding, infant formula supplementation, and Autistic Disorder: the results of a parent survey. International breastfeeding journal. 2006; 1:16. [PubMed: 16978397]

56. Ledford JR, Gast DL. Feeding problems in children with autism spectrum disorders: A review. Focus Autism Other Dev Disabl. 2006; 21:153-166.

57. Field D, Garland M, Williams K. Correlates of specific childhood feeding problems. Journal of paediatrics and child health. 2003; 39:299-304. [PubMed: 12755939]

58. Sharp WG, Jaquess DL, Luckens CT. Multi-method assessment of feeding problems among children with autism spectrum disorders. Res Autism Spectr Disord. 2013; 7:56-65.

59. Hediger ML, et al. Reduced bone cortical thickness in boys with autism or autism spectrum disorder. Journal of autism and developmental disorders. 2008; 38:848-856. [PubMed: 17879151]

60. Bandini LG, et al. Food selectivity in children with autism spectrum disorders and typically developing children. The Journal of pediatrics. 2010; 157:259-264. [PubMed: 20362301]

61 . Zimmer MH, et al. Food variety as a predictor of nutritional status among children with autism. Journal of autism and developmental disorders. 2012; 42:549-556. [PubMed: 21556968]

62. Huncharek M, Kupelnick B. Dietary fat intake and risk of epithelial ovarian cancer: a metaanalysis of 6,689 subjects from 8 observational studies. Nutrition and cancer. 2001; 40:87-91. [PubMed: 11962260] 
63. Mente A, de Koning L, Shannon HS, Anand SS. A systematic review of the evidence supporting a causal link between dietary factors and coronary heart disease. Archives of internal medicine. 2009; 169:659-669. [PubMed: 19364995]

64. Sharp WG, Jaquess DL, Morton JF, Herzinger CV. Pediatric feeding disorders: a quantitative synthesis of treatment outcomes. Clinical child and family psychology review. 2010; 13:348-365. [PubMed: 20844951]

65. Buie T, et al. Evaluation, diagnosis, and treatment of gastrointestinal disorders in individuals with ASDs: a consensus report. Pediatrics. 2010; 125 (Suppl 1):S1-18. [PubMed: 20048083]

66. Ibrahim SH, Voigt RG, Katusic SK, Weaver AL, Barbaresi WJ. Incidence of gastrointestinal symptoms in children with autism: a population-based study. Pediatrics. 2009; 124:680-686. [PubMed: 19651585]

67. Matson JL, Fodstad JC. The treatment of food selectivity and other feeding problems in children with autism spectrum disorders. Research in Autism Spectrum Disorders. 2009; 3:455-461.

68. Ahearn WH, Castine T, Nault K, Green G. An assessment of food acceptance in children with autism or pervasive developmental disorder-not otherwise specified. Journal of autism and developmental disorders. 2001; 31:505-511. [PubMed: 11794415]

69. Williams BL, et al. Impaired carbohydrate digestion and transport and mucosal dysbiosis in the intestines of children with autism and gastrointestinal disturbances. PloS one. 2011; 6:e24585. [PubMed: 21949732]

70. Piazza CC, et al. Functional analysis of inappropriate mealtime behaviors. Journal of applied behavior analysis. 2003; 36:187-204. [PubMed: 12858984]

71. Nikolov RN, et al. Gastrointestinal symptoms in a sample of children with pervasive developmental disorders. Journal of autism and developmental disorders. 2009; 39:405-413. [PubMed: 18791817]

72. Whorwell PJ, et al. Efficacy of an encapsulated probiotic Bifidobacterium infantis 35624 in women with irritable bowel syndrome. The American journal of gastroenterology. 2006; 101:1581-1590. [PubMed: 16863564]

**73. Rhee SH, Pothoulakis C, Mayer EA. Principles and clinical implications of the brain-gut-enteric microbiota axis. Nat Rev Gastroenterol Hepatol. 2009; 6:306-314. An excellent overview of the cellular and biochemical pathways of interaction among the gut, brain, and microbiome. [PubMed: 19404271]

74. Mayer EA. Gut feelings: the emerging biology of gut-brain communication. Nat Rev Neurosci. 2011; 12:453-466. [PubMed: 21750565]

75. Sudo N. Role of microbiome in regulating the HPA axis and its relevance to allergy. Chem Immunol Allergy. 2012; 98:163-175. [PubMed: 22767063]

76. Round JL, Mazmanian SK. The gut microbiota shapes intestinal immune responses during health and disease. Nat Rev Immunol. 2009; 9:313-323. [PubMed: 19343057]

77. Diaz Heijtz R, et al. Normal gut microbiota modulates brain development and behavior. Proc Natl Acad Sci U S A. 2011; 108:3047-3052. [PubMed: 21282636]

78. Clarke G, et al. The microbiome-gut-brain axis during early life regulates the hippocampal serotonergic system in a sex-dependent manner. Mol Psychiatry. 2012

79. Bercik P, et al. The intestinal microbiota affect central levels of brain-derived neurotropic factor and behavior in mice. Gastroenterology. 2011; 141:599-609. 609 e591-593. [PubMed: 21683077]

80. Tang X, Orchard SM, Sanford LD. Home cage activity and behavioral performance in inbred and hybrid mice. Behav Brain Res. 2002; 136:555-569. [PubMed: 12429418] 